Bernhard, S. S. R.; Locke, G. M.; Plunkett, S.; Meindl, A.; Flanagan, K. J.; Senge, M. O. (2018): Cubane Cross-Coupling and Cubane-Porphyrin Arrays.

Chemistry - A European Journal 24, 1026-1030.

\title{
Cubane Cross-Coupling and Cubane-Porphyrin Arrays
}

\author{
Stefan S. R. Bernhard, Gemma M. Locke, Shane Plunkett, Alina Meindl, Keith J. Flanagan and \\ Mathias O. Senge* \\ Dedicated to Prof. Dr. Dr. h.c. Henning Hopf
}

\begin{abstract}
The (im)possible Cube - Herein an improved methodology for aryl-cubane cross-coupling is reported. The peculiarities of the cubane core and its behavior during crosscoupling conditions are analyzed, while the versatility of this adapted Baran cross-coupling methodology is demonstrated by the synthesis of various aryl-cubane systems, among them coupling products of cubanes and porphyrins. Furthermore arm extension of alkynylcubanes via Sonogashira reactions is demonstrated, showcasing the first proof of the stability of the cubane core in the presence of palladium-catalysts.
\end{abstract}

Even 50 years after its first synthesis by Eaton et al. in 1964, the chemistry of cubane still presents a synthetic challenge and is underdeveloped, especially in terms of modern cross-coupling methodologies ${ }^{[1]}$ Most of the derivatizations arise from various conventional functional group interconversions of the dimethylcubane-1,4-dicarboxylate. Current state of the art is almost solely the use of "classical" carbonyl chemistry, ${ }^{[2]}$ thus limiting the scope of potential cubane compounds and therefore access to advanced cubane systems. Just recently cubanes began to draw attention again due to their biological properties and their use as a benzene bioisostere..$^{[3]}$ Our interest in cubane arose from two different origins. Firstly, we recognized the lack of useful reactions to install aryl groups directly onto the cubane core. Secondly, we were interested in utilizing cubanes as a rigid linker unit for applications in materials chemistry. However to achieve this, the right tools had to be developed, tools outside the "carbonyl-world". Screening the literature revealed only four different approaches, each one with their own significant drawbacks (Scheme 1).

The first example of cubane-aryl systems was mentioned by Moriarty et al. in 1989 describing the radical functionalization of cubane carboxylic acid in the presence of $\mathrm{Pb}(\mathrm{OAc})_{4}$ and benzene. ${ }^{[4]}$ Even though the yield is high, this method is only suitable for a limited number of aryl groups and suffers from poor regioselectivity. Eaton's group utilized the highly reactive cubane-1,4-diyl diradical for phenylation, in order to prepare cubane rods in "living polymerization"-like fashion. ${ }^{[5]}$ Here the major disadvantage is the missing functional group tolerance due to harsh conditions. Recently, the Baran group has shown the versatility of their newly developed redox-active ester concept, proving the robustness of their method by preparing mono- and diarylated cubanes shortly after. ${ }^{[6]}$ Thus far, this

[a] Dr. S. S. R. Bernhard, G. M. Locke, Dr. S. Plunkett, A. Meindl, Keith J. Flanagan, Prof. Dr. M. O. Senge School of Chemistry, SFI Tetrapyrrole Laboratory Trinity College Dublin, The University of Dublin, Trinity Biomedical Sciences Institute, 152-160 Pearse Street, Dublin 2, (Ireland) E-mail: sengem@tcd.ie

Supporting information for this article is given via a link at the end of the document. approach represents the benchmark in terms of flexibility, but again only results in low yields, even for simple aryl substrates.

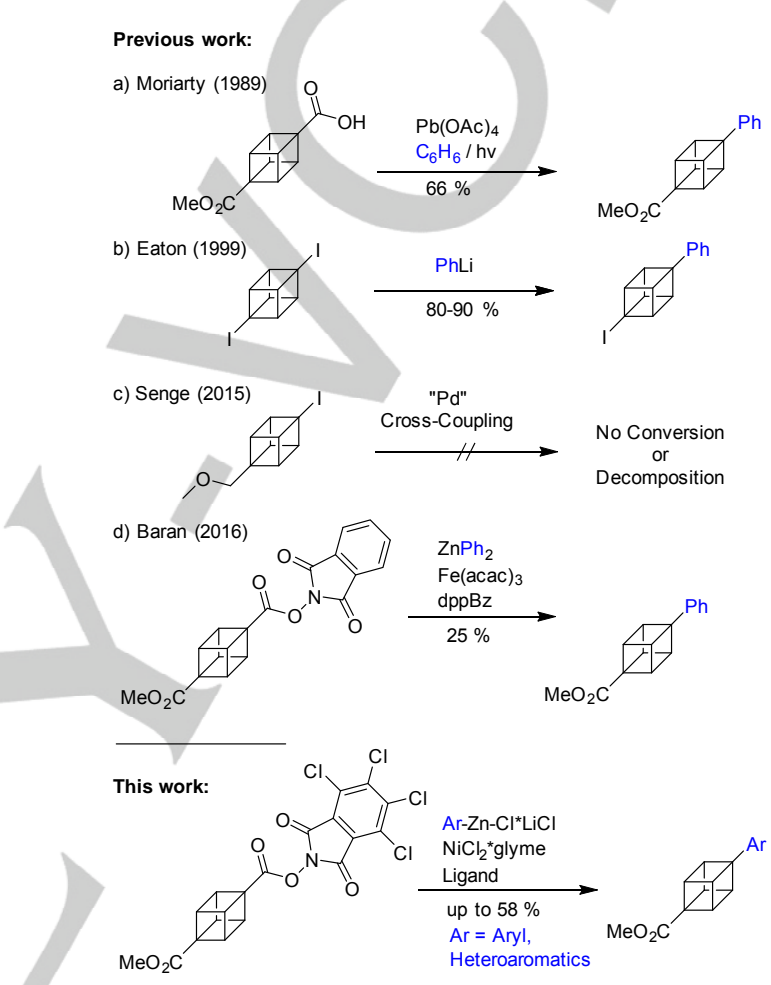

Scheme 1. State-of-the-art of cubane-aryl coupling.

Our own initial approach for applying palladium catalyzed crosscoupling chemistry on cubanes, failed, unfortunately, owing to the instability of the cubane core in presence of palladium. ${ }^{[7,8]}$ With this knowledge we turned our attention to a single electron transfer mechanism (SET), circumventing the requirement of the oxidative addition of the transition metal to the cubane core. Even though the first approach via an iron-catalyzed Kumada coupling proved unsuccessful, ${ }^{[9]}$ we were inspired by the versatility of redox-active esters for decarboxylative cubane-aryl cross-coupling (Baran/Weix coupling). ${ }^{[10]}$ The reported yield of $25 \%$ for the iron-catalyzed coupling ${ }^{[6]}$ raised our curiosity to identify the key obstacles for the cubane-aryl coupling. A closer look revealed five major contributing factors i) solvent, ii) temperature, iii) concentration, iv) $\mathrm{Ni}$-source, and v) ligand (Scheme 2).

After thorough investigation of the Baran cross-coupling methodology, the influence of the ligand was identified as the key parameter ${ }^{[11]}$ Ligand design is a powerful tool to modulate the reactivity of transition metal catalysts and to control the reaction pathway. Ligand redox effects play an important role during the catalytic cycle. ${ }^{[12]}$ Within the tested library of bipyridines, bisphosphines and tridentate ligands, the very electron-deficient 4,4'-functionalized bipyridines outperformed 
other ligand systems; especially the bisphosphine and rigid phenanthroline ligands. According to our findings the ideal ligand possesses some flexibility along the biaryl axis, has no substituent in the a-position to the nitrogen and carries electron withdrawing substituents in the 4,4'-positions. We hypothesize the influence of the electron-poor $4,4^{\prime}$-position to affect the rate determining step of the catalytic cycle, namely the reductive elimination of the cubane and aryl residue from the nickel center. Bulky ligands (dtbbpy, L1) and electron withdrawing substituents (L2) favor the reductive elimination due to strain release and electronic stabilization. ${ }^{[13]}$ This assumption is in accordance with previous findings of cubane's reactvity: i) the cubyl radical is rather stable ${ }^{[14]}$ and is captured in the presence of Michael acceptors or radical scavengers (e.g., TEMPO); ${ }^{[15]}$ ii) the reductive elimination proceeds faster for hydrides and the according hydride-coupling product can be obtained in excellent yield. ${ }^{[16,17]}$
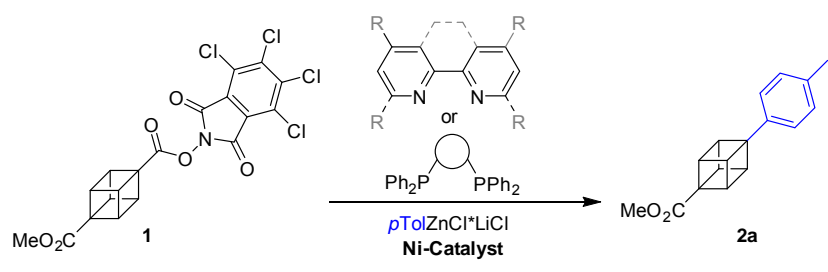

Optimization
- Solvent
- Temperature
- Concentration
- Ni catalyst
- Ligand

- Polar environment favorable Broad temperature range - High concentration favorable Decisive influence

$\begin{array}{ll} & \text { Conditions } \\ & -\mathrm{DMF} / \mathrm{THF} 3 / 2 \\ \square & -0{ }^{\circ} \mathrm{C}-40^{\circ} \mathrm{C} \\ & -\mathrm{C}=0.05-0.06 \mathrm{mmol} / \mathrm{ml} \\ & -\mathrm{NiCl}{ }_{2}^{*} \text { glyme } \\ & - \text { Electron-poor ligands }\end{array}$

Scheme 2. Contributing factors influencing cubane cross-couplings

Realizing the impediments and to test our hypothesis we investigated the scope of our new catalyst/ligand systems (Scheme 3). The coupling of very electron-rich aryl moieties proceeded in good yields of over $50 \%$ (2d, 2f, $2 \mathbf{2})$. Likewise, unfunctionalized aryl groups could be coupled smoothly $(\mathbf{2 a}, \mathbf{2 b}$, 2c). One remaining problem is the coupling of electronic deficient aryl substrates. A weak electron withdrawing substituent in the $p$-position is tolerated, but only with a significant decrease in yield $(\mathbf{2 j}, 18 \%)$.

In case of very electron-poor aryl groups and heteroaromatic systems, an electron-richer ligand (L1) proved to be beneficial in order to overcome decomposition before the coupling $(2 \mathrm{~m}, \mathbf{2 n}$, 20). Generally, the yield is lower for these important classes of compounds, but we were pleased to observe the first directly linked cubane-porphyrin system $(2 \mathrm{~m}, \mathbf{2 n}, \mathbf{2 0})$, a system which has not been reported before. ${ }^{[18]}$ The preparation of a suitable porphyrin-zinc species was achieved by adapting the iodinemetal exchange developed by the Osuka group. ${ }^{[19,20]}$

Notable is the smooth coupling of useful compound $\mathbf{2 f}$, which can be subject of further functionalization and was used for the preparation of cubane-linked porphyrin-system 10a (vide infra). In addition, the procedure could be used to prepare difunctionalized aryl-cubane $\mathbf{2} \mathbf{h}$ from $\mathbf{2 a}$ using a sequence of deprotection of the methyl ester, activation of the carboxylic acid, and Ni-catalyzed coupling. A major limitation right now is to overcome the sterical hindrance of ortho-substituents (entry $\mathbf{2 e}$, $25 \%$ ) and to extend the scope for further heteroaromatic coupling partners. With the direct-coupled cubane-aryl systems in hand we decided to go back to our initial approach of palladium-catalyzed cross-coupling chemistry with cubanes. ${ }^{[8]}$
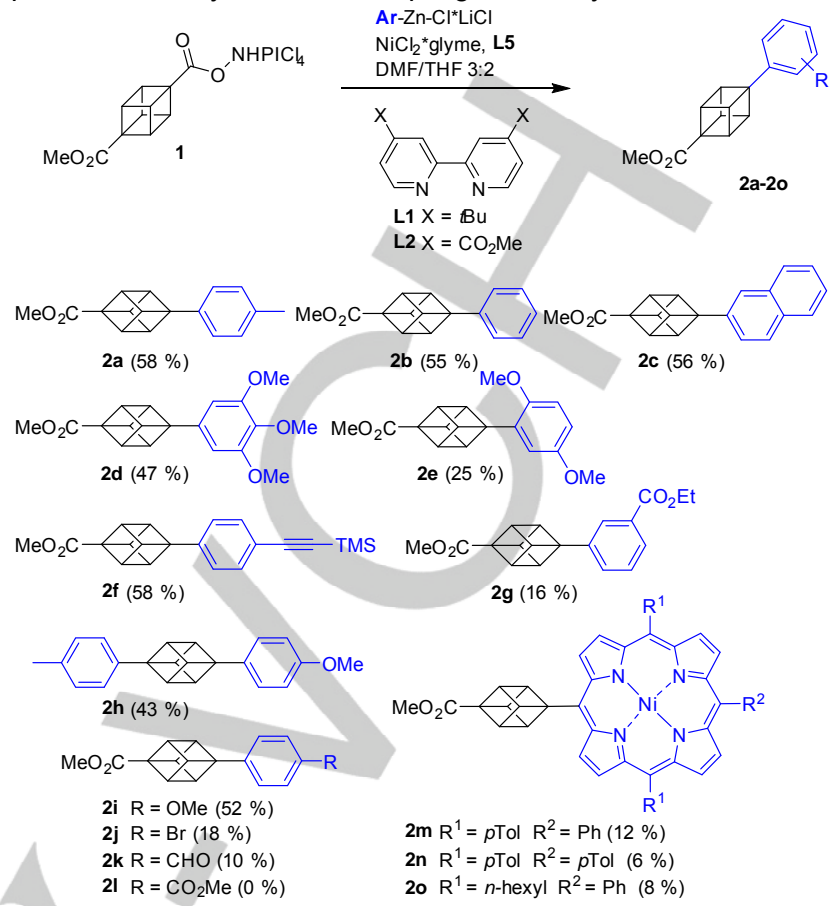

Scheme 3. Nickel-catalyzed cross-coupling and substrate scope.

Since the direct approach proved to be unfruitful, we decided to apply an arm extension of the cubane core with alkynyl-groups, hoping that the palladium/copper-alkynyl-cubane intermediate is more stable than the directly attached counterparts. To our delight the initial reaction showed clean conversion of the cubane alkynyl 3 to the substituted product (Table 1). ${ }^{[21]}$

Table 1. Substrate scope for palladium-catalyzed Sonogashira coupling of alkynyl cubane 3.

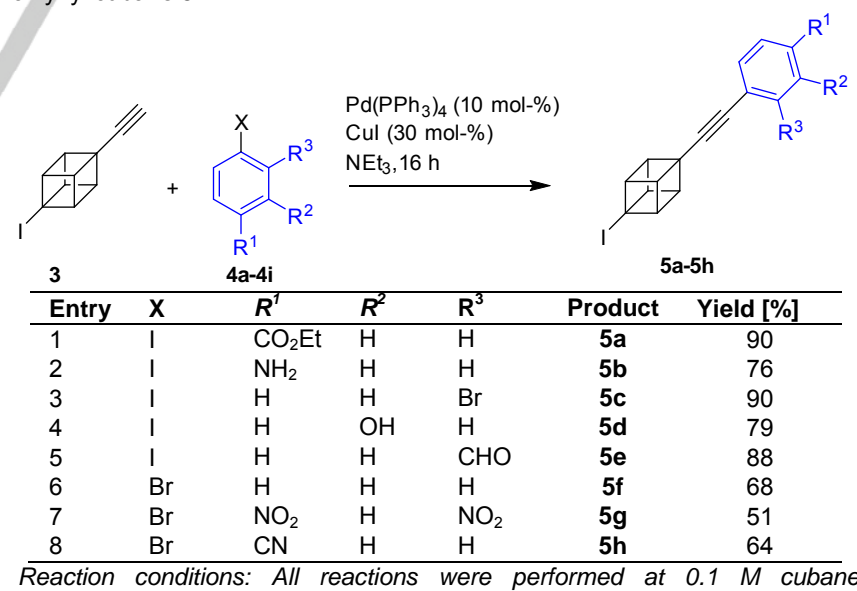
concentration using 3 equivalents of aryl halide, $\mathrm{Pd}\left(\mathrm{PH}_{3}\right)_{4}(10 \mathrm{~mol}-\%)$ and $\mathrm{Cu}$ (30 mol-\%) under argon for $16 \mathrm{~h}$. Where applicable, yields are isolated yields after chromatography on silicia gel.

After optimization of the general coupling conditions, we were interested to see, if the Sonogashira reaction was tolerant to electronic changes in the aryl substituent. The presence of electron-withdrawing groups on the aryl iodides in $p$ - or $o$ positions (entries 1,3,5) led to product formation in excellent yields $(\sim 90 \%)$. With the introduction of electron donating groups (entries 2,4$)$ a slight decrease in yield was observed $(76-80 \%)$. 
This behavior is in accordance with the literature, as oxidative addition of the aryl halide to the active palladium species is believed to be the rate-limiting step. ${ }^{[2]}$ Less reactive aryl bromides required more forcing conditions and only electronpoor aromatic systems (entries 6-8) gave product formation. Particularly product $\mathbf{5 g}$ may be highlighted as belonging to a new family of nitro-cubanes. The ease of attachment of two nitro-groups in the direct proximity to the alkynyl-cubane-core could give a new boost to the search for cheaper cubane-based high energy materials. ${ }^{[23]}$

The stability of alkynyl cubanes towards palladium and copper catalysis during Sonogashira-conditions is astonishing as previous findings showed that the presence of $\mathrm{Rh}(\mathrm{I})$ leads to a rearrangement of the alkynyl-cubanes to either cyclooctatetraen derivatives or tricyclooctadiene. ${ }^{[24]}$ In addition, the presence of $\mathrm{Pd}$ on charcoal either leads to a ring-opening to cyclooctatetraen, ${ }^{[25]}$ or to reduction to bicyclo[2.2.2]-octane under hydrogen atmosphere. ${ }^{[26]}$ However, with this reassuring indication of cubane's stability, we turned our interest to more delicate cubane-porphyrin systems (Scheme 4).

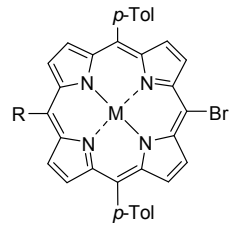

$6 \mathbf{a}(\mathrm{M}=2 \mathrm{H}, \mathrm{R}=\mathrm{Ph})$

$6 c(M=2 H, R=B r)$
$6 \mathrm{~b}(\mathrm{M}=\mathrm{Ni}(\mathrm{II}), \mathrm{R}=\mathrm{Ph})$

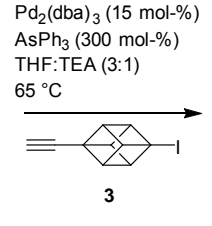

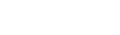

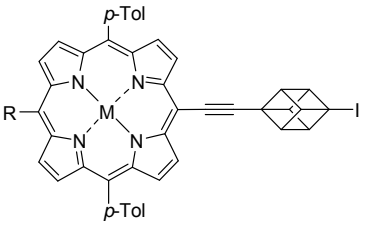

$7 \mathrm{a}(\mathrm{M}=2 \mathrm{H}, \mathrm{R}=\mathrm{Ph}), 73 \%$ $7 b(M=N i(I), R=P h), 63 \%$

$7 c(M=2 H, R=$
Figure 1. Molecular structure of the porphyrin-alkynyl-cubane $\mathbf{7 b}$ showing top and side views (thermal displacement $50 \%$ ).

Adjustment of the conditions and utilization of a Cu-free methodology provided us with arm-extended porphyrin-cubane systems $7 \mathbf{a}(73 \%)$ and $\mathbf{7 b}(63 \%)$ in good yields (Figure 1). ${ }^{[28]}$ Furthermore, this approach was extended to dibrominated-5,15porphyrin $6 \mathbf{c}$, to achieve a linear porphyrin with two cubane handles on each side in $7 c(51 \%) .{ }^{[2]}$ Even more delicate porphyrin-cubane systems were achieved by using the bisalkynyl-cubane, either as mono-protected $\mathbf{8 a}$ or as the bisalkynyl-species $\mathbf{8 b}$ (Table 2). ${ }^{[21]}$

Table 2. Sonogashira coupling of bisalkynyl-cubanes with bromoporphyrins.

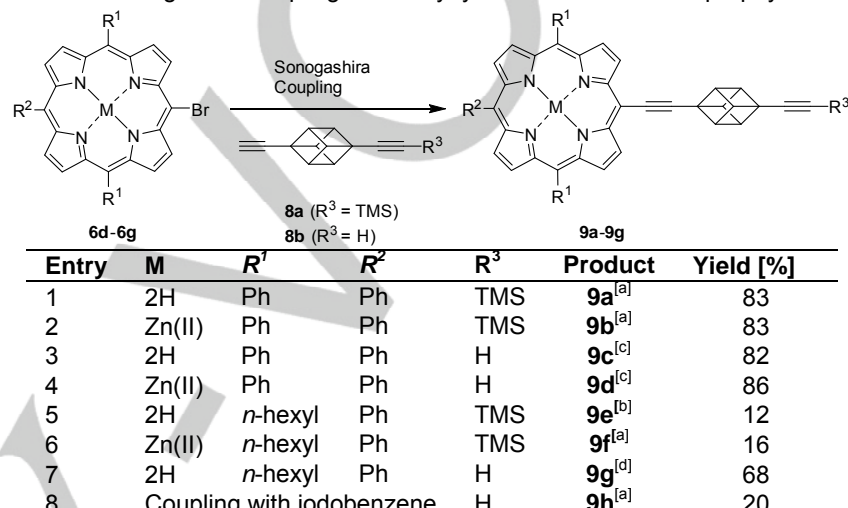
Reaction conditions: All reactions were performed with $0.33 \mathrm{mmol}$ of cubane $\mathbf{8 a} / \mathbf{8 b}$ and $0.29 \mathrm{mmol}$ porphyrin $\mathbf{6 d - 6 g}$ at $65^{\circ} \mathrm{C}$ for $3 \mathrm{~h}$ with either $[\mathrm{a}] \mathrm{Pd}\left(\mathrm{Ph}_{3}\right)_{4}$ (10 mol-\%, Cul (20 mol-\%), THF/TEA (10:3) or [b] $\mathrm{Pd}_{2}(\mathrm{dba})_{3}(15 \mathrm{~mol}-\%)$, $\mathrm{AsPh}_{3}$ (30 mol-\%), THF/TEA (3:1). Deprotection of the of according TMSprotected coupling product were performed with either [c] TBAF/THF or [d] $\mathrm{K}_{2} \mathrm{CO}_{3} / \mathrm{MeOH}$.

Scheme 4. Cubane-porphyrin systems via Sonogashira coupling.

The transfer of the optimized conditions for Sonogashira coupling for normal aryl substrates was not effective in the initial reaction. Next to insertion of copper into the macrocyclic porphyrin core we observed the formation of the Glaser-coupled cubane-dimer, rather than the Sonogashira product. ${ }^{[27]}$

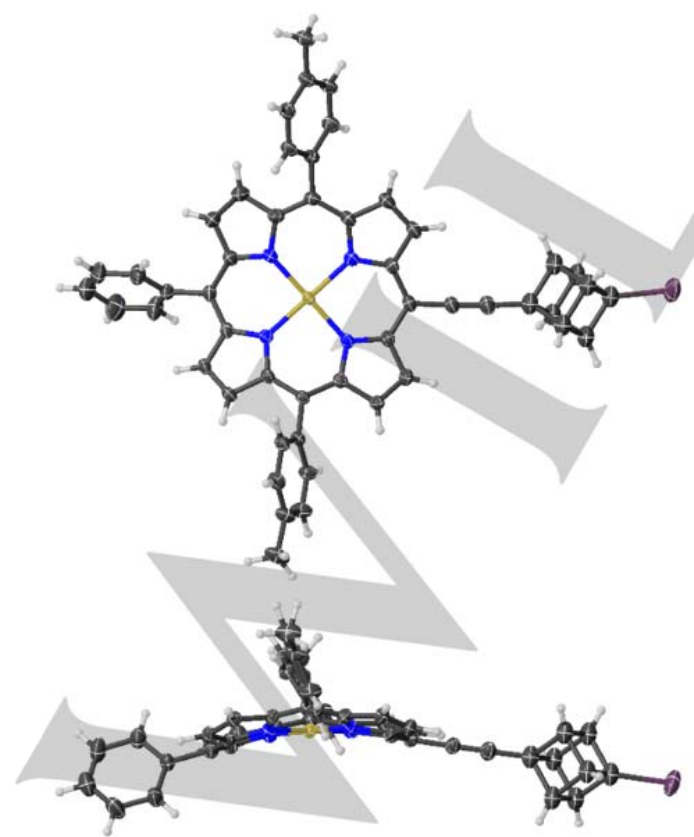

To overcome the low solubility of the triphenylporphyrins $\mathbf{6 d - 6 e}$, we changed to more soluble dialkylporphyrins $6 f-6 g$, but the yield of the isolated pure compound dropped significantly. ${ }^{[30]}$ Thus far, the limitation for this kind of systems is the preparation of bisporphyrin-alkynyl-cubanes, which could not be isolated. ${ }^{[11]}$ Interestingly, the instability of the material arises from the porphyrin-fragment rather than the cubane-system, since a double Sonogashira coupling of $\mathbf{8} \mathbf{b}$ with excess of iodobenzene yielded the diarylated-product $9 \mathrm{~h}$ without notable decomposition of the cubane core (entry 8 ).

Finally, we were able to apply the methodology presented for the synthesis of a cubane-linked porphyrin 10a (Figure 2).

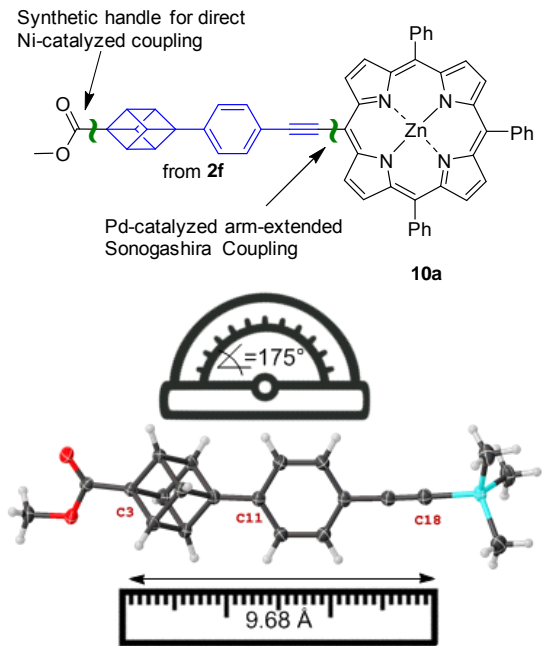


Figure 2. A series of linear linkers: Cubane-linked porphyrin 10a and molecular structure of precursor $\mathbf{2 f}$ (thermal displacement $50 \%$ ).

The synthesis was achieved with an overall yield of $20 \%$, starting from building block $2 \mathrm{f}$ with deprotection of the alkynyl followed by $\mathrm{Pd}$-catalyzed coupling with bromoporphyrin $6 \mathrm{e}^{[32]}$ Porphyrin 10a is the first example of a cubane-linked porphyrin array with a further synthetic handle, which represents a significant step towards using cubane-linked systems as electron transfer compounds with rigid resistor units. ${ }^{[33]}$ With a dimension of $9.68 \AA$ from one cubane-carbon (C3) to the end of the acetylen-unit $(\mathrm{C} 18)$ and with all carbons in line $\left({ }_{4} \mathrm{C}_{3} \mathrm{C}_{11} \mathrm{C}_{18}=175^{\circ}\right)$ this represents an interesting new class of linker system.

In conclusion, a modified cubane-aryl coupling via decarboxylative Baran coupling is reported. After systematic investigations the key obstacle of cubane cross-coupling was identified and circumvented by altering the electron density of the transition metal through an adjusted choice of ligand. Furthermore, for the first time not only the preparation of directly linked cubane-porphyrin system was achieved but also the possibility of Sonogashira functionalization of alkynyl armextended cubanes, proving the general compatibility of cubanes and Pd-catalyzed cross-coupling reactions. These crosscoupling reactions allow the preparation of new classes of cubane compounds, important not only for medicinal but also for materials chemistry. Further investigations on the unique behavior of cubane during (radical) cross-coupling conditions and the exact mechanism of decomposition are the topic of ongoing investigations.

\section{Acknowledgements}

This work was supported by grants from the Science Foundation Ireland (SFI IVP 13/IA/1894) and the Irish Research Council (GOIPG/2015/3700).

\section{Keywords: Cubanes $\cdot$ Cross-Coupling $\cdot$ Porphyrins $•$} Sonogashira Coupling $\bullet$ Nickel Catalysis

[1] a) P. E. Eaton, T. W. Cole, J. Am. Chem. Soc. 1964, 86, 962-964; b) P. E. Eaton, T. W. Cole, J. Am. Chem. Soc. 1964, 86, 3157-3158.

[2] Examples for recent cubane-carbonyl functional group interconversions a) K. C. Nicolaou, D. Vourloumis, S. Totokotsopoulos, A. Papakyriakou, H. Karsunky, H. Fernando, J. Gavrilyuk, D. Webb, A. F. Stepan, ChemMedChem 2016, 11, 31-37; b) J. Wlochal, R. D. M. Davies, J. Burton, Org. Lett. 2014, 16, 4094-4097; general aspects of cubane chemistry: c) K. J. Biegasiewicz, J. R. Griffiths, G. P. Savage, J. Tsanaktsidis, R. Priefer, Chem. Rev. 2015, 115, 6719-6745.

[3] a) P. E. Eaton, Angew. Chem. Int. Ed. Engl. 1992, 31, 1421-1436 Angew. Chem, 1992, 104, 1447-1462; b) B. A. Chalmers, H. Xing, S. Houston, C. Clark, S. Ghassabian, A. Kuo, B. Cao, A. Reitsma, C. P. Murray, J. E. Stok, G. M. Boyle, C. J. Pierce, S. W. Littler, D. A. Winkler, P. V. Bernhardt, C. Pasay, J. J. De Voss, J. McCarthy, P. G. Parsons, G. H. Walter, M. T. Smith, H. M. Cooper, S. K. Nilsson, J. Tsaknaktsidis G. P. Savage, C. M. Williams, Angew. Chem. Int. Ed. 2016, 55, 35803585; Angew, Chem. 2016, 128, 3644-3649.

[4] R. M. Moriarty, J. S. Khosrowshahi, J. Am. Chem. Soc. 1989, 111, 8943-8944.

[5] P. E. Eaton, K. Pramod, T. Emrick, R. Gilardi, J. Am. Chem. Soc. 1999 121, 4111-4123.

[6] F. Toriyama, J. Cornella, L. Wimmer, T. Chen, D. D. Dixon, G. Creech, P. S. Baran, J. Am. Chem. Soc. 2016, 138, 11132-11135.
[7] a) Instability of cubane towards rhodium: L. Cassar, P. E. Eaton, J. Halpern, J. Am. Chem. Soc. 1970, 92, 3515-3518; b) Instability towards silver and palladium P. E. Eaton, L. Cassar, J. Halpern, J. Am. Chem. Soc. 1970, 92, 6366-6368.

[8] S. Plunkett, K. J. Flanagan, B. Twamley, M. O. Senge, Organometallics 2015, 34, 1408-1414.

[9] For further details see SI (S4). The attack into the outer $\pi^{*}$-orbital of the redox-active phthalimide moiety further away from the bulky cubane core seems more promising than the radical generation from the according cubane-iodines in the Kumada coupling.

[10] a) J. Cornella, J. T. Edwards, T. Qin, S. Kawamura, J. Wang, C. Pan, R. Gianatassio, M. Schmidt, M. D. Eastgate, P. S. Baran, J. Am. Chem. Soc. 2016, 138, 2174-2177. b) K. M. M. Huihui, J. A. Caputo, Z. Melchor, A. M. Olivares, A. M. Spiewak, K. A. Johnson, T. A DiBenedetto, S. Kim, L. K. G. Ackerman, D. J. Weix, J. Am. Chem. Soc. 2016, 138, 5016-5019.

[11] Full details in SI (S5-S7)

[12] a) G. D. Jones, J. L. Martin, C. McFarland, O. R. Allen, R. E. Hall, A. D. Haley, R. J. Brandon, T. Konovalova, P. J. Desrochers, P. Pulay, D. A. Vivic, J. Am. Chem. Soc. 2006, 128, 13175-13183. b) E. C. Hansen, D. J. Pedro, A. C. Wotal, N. J. Gower, J. D. Nelson, S. Caron, D. J. Weix, Nat. Chem. 2016, 8, 1126-1130.

[13] D. A. Culkin, J. F. Hartwig, Organometallics 2004, 23, 3398-3416.

[14] J. C. Walton, Chem. Soc. Rev. 1992, 21, 105-122.

[15] In the presence of TEMPO no aryl-cubane coupling was observed, indicating a rather long living cubyl radical. However, isolation of the recombination product of TEMPO and cubane was not possible.

[16] T. Qin, L. R. Malins, J. T. Edwards, R. R. Merchant, A. J. E. Novak, J. Z Zhong, R. B. Mills, M. Yan, C. Yuan, M. D. Eastgate, P. S. Baran, Angew. Chem. Int. Ed. 2017, 56, 260-265; Angew. Chem. 2017, 129, 266-271

[17] J. J. Low, W. A. Goddard III, J. Am. Chem. Soc. 1984, 106, 8321-8322.

[18] Not only classical porphyrin condensations with cubane aldehydes and pyrrole but also nucleophilic additions of lithium cubanes to free mesoporphyrins failed (unpublished results).

[19] a) K. Fujimoto, H. Yorimitsu, A. Osuka, Eur. J. Org. Chem. 2014, 43274334; b) A. Krasovskiy, P. Knochel, Angew. Chem. Int. Ed. 2004, 43, 3333-3336; Angew. Chem. 2004, 116, 3396-3399.

[20] Despite careful control of temperature and concentrations, we observed a nucleophilic substitution at the meso-position, yielding in an isopropyl porphyrin. (See SI compound 2p, S20). Similar to the well-developed nucleophilic substitution of porphyrins with organolithium compounds: W. W. Kalisch, M. O. Senge, Angew. Chem. Int. Ed. Engl. 1998, 37, 1107-1109; Angew. Chem. 1998, 110, 1156-1159.

[21] Alkynylcubanes $\mathbf{3}, \mathbf{8} \mathbf{a}$ and $\mathbf{8 b}$ were prepared according to reference 24 For further details see SI (S8).

[22] K. Sonogashira, J. Organomet. Chem. 2002, 653, 46-49.

[23] a) P. E. Eaton, M. Zhang, R. Gilardi, N. Gelber, S. Iyer, R. Surapaneni, Propellants Explos. Pyrotech. 2002, 27, 1-6; b) P. E. Eaton, R. L. Gilardi, M. Zhang, Adv. Mater. 2000, 12, 1143-1148.

[24] P. E. Eaton, E. Galoppini, R. Gilardi, J. Am. Chem. Soc. 1994, 116 7588-7596.

[25] Q. I. Chruches, R. J. Mulder, J. M. White, J. Tsanaktsidis, P. J. Duggan, Aust. J. Chem. 2012, 65, 690-693.

[26] R. Stoper, H. Musso, E. Osawa, Tetrahedron 1986, 42, 1757-1761.

[27] a) C. Glaser, Justus Liebigs Ann. Chem. 1870, 154, 137-171. b) K. S, Sindhu, G. Anilkumar, RSC Adv. 2014, 4, 27867-27887.

[28] R. W. Wagner, T. E. Johnson, F. Li, J. S. Lindsey, J. Org. Chem. 1995 60, 5266-5273.

[29] For further details see SI (S9)

[30] A determination of the yield from the crude reaction mixture via ${ }^{1} \mathrm{H}-\mathrm{NMR}$ indicated yields comparable to the corresponding arylated-porphyrins (entry 1-4). Hence, decomposition during the purification is responsible for the low yields of the dialkyl-porphyrin systems. Only the reaction with the bisalkynyl $\mathbf{8 b}$ achieved the product in good yields again. Due to the high copper concentration during the reaction conditions, the insertion of copper was detected as side reaction yielding porphyrin 9 i.

[31] Isolation of the pure product from the reaction mixture was not achieved, either by column chromatography on silica gel, aluminium oxide, celite, size exclusion chromatography or by iterative recrystallization in various 
solvents. The crude purple solid appeared to be insoluble in most organic solvents and UV-vis studies indicated the absence of any porphyrinoid species.

[32] For further details see SI (S38-S39)

[33] G. M. Locke, M. O. Senge, ECS Trans. 2016, 72, 1-11. 


\section{COMMUNICATION}

\section{Alea lacta Est}

The compatibility of cubanes and transition metals (Ni, Pd) is explored and a direct arylation of cubanes via a Baran-type nickelcatalyzed radical cross-coupling and arm-extended Sonogashira coupling of alkynyl-cubanes is reported. Furthermore the first series of cubane-porphyrins is prepared.

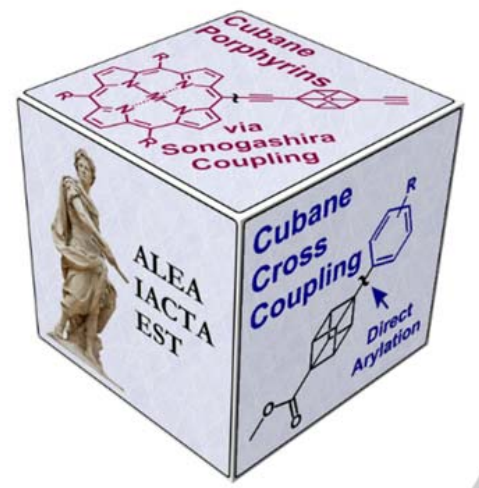

Stefan S. R. Bernhard, Gemma M. Locke, Shane Plunkett, Alina Meindl, Keith J. Flanagan and Mathias O. Senge*

Page No. - Page No.

Cubane Cross-Coupling and Cubane-Porphyrin Arrays 